\title{
Transfer of autocollimator calibration for use with scanning gantry profilometers for accurate determination of surface slope and curvature of state-of-the-art $x$-ray mirrors
}

\author{
Ian Lacey*a, Kevan Anderson ${ }^{\mathrm{a}}$, Jeff Dickert ${ }^{\mathrm{a}}$, Ralf D. Geckler ${ }^{\mathrm{b}}$, Andreas Just ${ }^{\mathrm{b}}$, Frank Siewert ${ }^{\mathrm{c}}$, \\ Brian V. Smith ${ }^{\mathrm{a}}$, and Valeriy V. Yashchuk ${ }^{\mathrm{a}}$ \\ ${ }^{a}$ Lawrence Berkeley National Laboratory, 1 Cyclotron Rd., Berkeley, California 94720; \\ ${ }^{\mathrm{b}}$ Physikalisch-Technische Bundesanstalt, Bundesallee 100, D-38116 Braunschweig, Germany; \\ ${ }^{c}$ Helmholtz Zentrum Berlin für Materialien und Energie, Institut für Nanometer Optik und \\ Technologie, Albert-Einstein-Str. 15, 12489 Berlin, Germany
}

\begin{abstract}
X-ray optics, desired for beamlines at free-electron-laser and diffraction-limited-storage-ring X-ray light sources, must have almost perfect surfaces, capable of delivering light to experiments without significant degradation of brightness and coherence. To accurately characterize such optics at an optical metrology lab, two basic types of surface slope profilometers are used: the long trace profilers (LTPs) and nanometer optical measuring (NOM) like angular deflectometers, based on electronic autocollimator (AC) ELCOMAT-3000. The inherent systematic errors of the instrument's optical sensors set the principle limit to their measuring performance. Where autocollimator of a NOM-like profiler may be calibrated at a unique dedicated facility, this is for a particular configuration of distance, aperture size, and angular range that does not always match the exact use in a scanning measurement with the profiler. Here we discuss the developed methodology, experimental set-up, and numerical methods of transferring the calibration of one reference AC to the scanning AC of the Optical Surface Measuring System (OSMS), recently brought to operation at the ALS X-Ray Optics Laboratory. We show that precision calibration of the OSMS performed in three steps, allows us to provide high confidence and accuracy low-spatial-frequency metrology and not 'print into' measurements the inherent systematic error of tool in use. With the examples of the OSMS measurements with a state-of-the-art x-ray aspherical mirror, available from one of the most advanced vendors of x-ray optics, we demonstrate the high efficacy of the developed calibration procedure. The results of our work are important for obtaining high reliability data, needed for sophisticated numerical simulations of beamline performance and optimization of beamline usage of the optics. This work was supported by the U. S. Department of Energy under contract number DE-AC02-05CH11231.
\end{abstract}

Keywords: synchrotron radiation, error suppression, calibration, metrology of x-ray optics, surface metrology, profilometry, DLSR, FEL

\section{INTRODUCTION}

X-ray optics, desired for the beamlines at free-electron-laser and diffraction-limited-storage-ring x-ray light sources, must have almost perfect surfaces, capable of delivering light to experiments without significant degradation of brightness and coherence [1,2]. Thus, beamlines, to be created or upgraded in the Advanced Light Source Upgrade (ALS-U) project [3,4], require x-ray optics with the residual (after subtraction of an ideal shape) shape errors of $<100 \mathrm{nrad}$ (root-mean-square, rms) in slope and $<2 \mathrm{~nm} \mathrm{rms}$ in height. In order to ensure the optimal usage of the superhigh-quality optics at the ALS-U, the dedicated ex situ metrology tools have to be capable of surface measurements with high accuracy and reliability, ideally, better than the specification by a factor of few (2-5).

To accurately characterize such optics at an optical metrology lab, two basic types of surface slope profilometers are used: the Long Trace Profilers (LTPs) [5-31] and Nanometer Optical Measuring Machine (NOM, [32-34]) like angular deflectometers [35-52], based on electronic autocollimator (AC) ELCOMAT-3000 [53]. The inherent systematic errors of the instrument's optical sensors set the principle limit to their measuring performance.

*ILacey@lbl.gov; phone 1510 495-2644; fax 1510 486-7696; https://als.lbl.gov/people/ian-lacey/ 
Where AC of a NOM-like profiler may be calibrated at a unique dedicated facility, for example, at the PhysikalischTechnische Bundesanstalt (PTB), Germany, using their sophisticated experimental method and set-up [54-56], this is for a particular configuration of distance, aperture size, and angular range that does not always match the exact use in a scanning measurement with the profiler.

Here, we discuss the developed experimental set-up, methodology, and numerical methods of transferring the calibration of one reference AC, performed at the PTB, to the scanning AC of the Optical Surface Measuring System (OSMS) [49-52], recently brought to operation at the ALS X-Ray Optics Laboratory (XROL) [57,58]. We show that precision calibration of the OSMS in-situ, with an integrated calibration system, allows us to provide high confidence and accuracy low-spatial-frequency metrology and not 'print into' measurements the inherent systematic error of tool in use. With the example of the OSMS measurements with a highly curved aspherical mirror with a length of the clear aperture of $600 \mathrm{~mm}$, available from one of the most advanced vendor of x-ray optics, we demonstrate the high efficacy of the developed calibration methodology and experimental methods. We believe that the results of our work are important for obtaining high reliability data, needed for sophisticated numerical simulations of beamline performance and optimization of beamline usage of the optics. The developed approaches are rather universal and, therefore, widely applicable.

\section{EXPERIMENTAL SET-UP OF THE ALS OSMS}

Figure 1 shows the XROL OSMS experimental set-up $[49,50]$ based on the multifunctional translation system, including 2D gantry and a custom tilt-flip-Z-translation stage (TFZS) and four electronic autocollimators ELCOMAT-3000 [53]. The AC in the surface-under-test (SUT) measuring (sample) channel, AC-1, is placed vertically on the OSMS X-axis translation carriage. In the course of measurements, the carriage with AC-1 is translated along the SUT, keeping the distance between the AC and the SUT practically unchanged. This design [38] allows a reliable application of the AC calibration performed at the same AC-to-SUT distance. Additionally, the problem of air convection noise [18] in the sample channel is solved with a close tube of the aperture system that shields the AC-1 light beam optical path. The payment for these advantages is a necessity to control the wobbling error with an additional AC, mounted on the righthand granite stand, AC-2 in Fig. 1. The air convection noise problem, transferred now to the reference channel, is overcome by appropriately filtering the reference data as discussed in detail in Ref. [50].

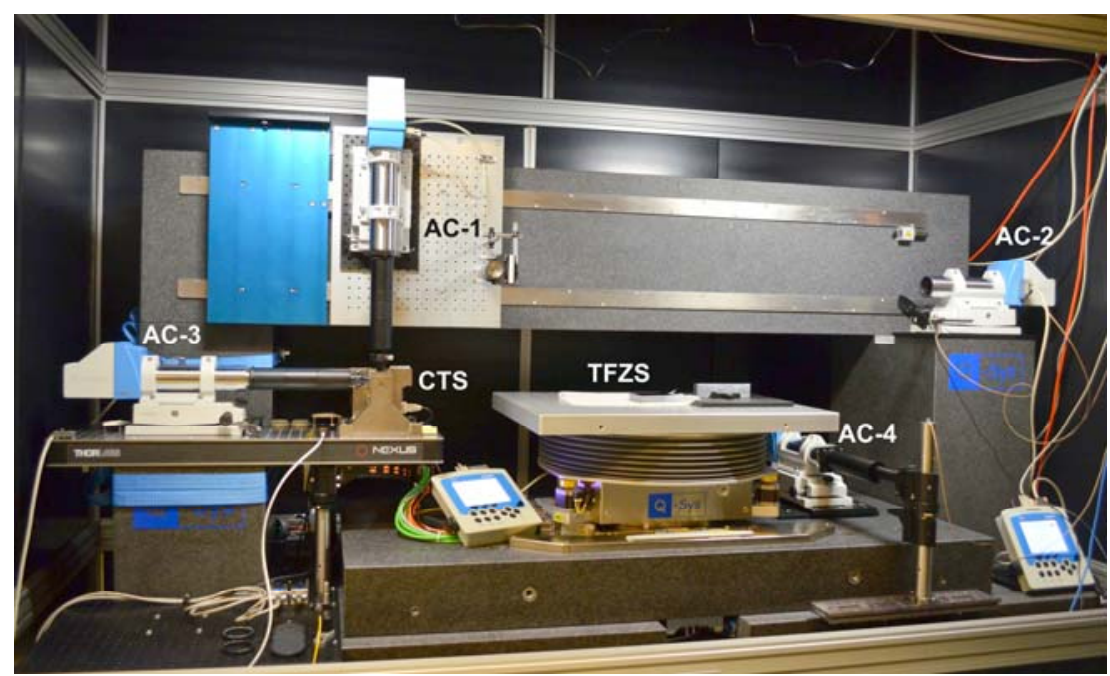

Figure 1. Experimental arrangement of the ALS XROL OSMS $[49,50]$ based on the multifunctional translation system, including 2D gantry and custom tilt-flip-Z-translation stage (TFZS), and four electronic autocollimators ELCOMAT-3000 [53], with a fixed distance AC-1 in the sample arm [38]. AC-2 monitors the X-axis carriage wobbling error. AC-3 serves as a transferable reference for in-situ angular calibration of AC-1. When the calibration tilt stage (CTS) removed, AC-3 can also be used as a constant-distance slope sensor for measurements with optics in the side-facing orientation. In this case, $\mathrm{AC}-4$, mounted to the $\mathrm{Y}$-axis movable slab, is used for monitoring the yaw-angle translation error of the slab.

To the best of our knowledge, the arrangement of an AC-based surface slope profilometer with a movable, vertically oriented $\mathrm{AC}$ and an additional $\mathrm{AC}$ in the reference channel was firstly considered in Ref. [38] and implemented and published in Refs. [47,49]. 
In the current arrangement of the XROL OSMS in Fig. 1, the autocollimator AC-3, precisely calibrated at the PTB [52], serves as a transferable reference for in-situ angular calibration of AC-1. Other indefeasible part of the calibration system (see Sec. 3) is a precision calibration-tilting-stage (CTS in Fig. 1) [59], designed and fabricated as a key element of the universal test mirror (UTM) system [60].

When the CTS removed, AC-3 can also be used as a constant-distance slope sensor for direct (without additional 90degree folding mirror or pentaprism) measurements with optics in the side-facing orientation. In this case, the $\mathrm{Y}$-axis slab of the OSMS gantry is translated, and AC-4, mounted to the movable slab, is used in the Y-axis reference channel for monitoring the yaw-angle translation error of the slab.

With the integrated custom tilt-flip-Z-translation stage (TFZS in Fig. 1), the OSMS is capable of fully automatic twodimensional (2D) surface slope mapping with tilting and flipping the SUT, realizing the advanced-optimal-scanningstrategy (AOSS) method for suppression of the instrumental systematic and temporal drift errors [22,51]. As demonstrated in Refs. [61] and Sec. 5, below, with the careful calibration of the AC sensors, the OSMS is capable for surface slope metrology of significantly curved aspherical x-ray mirrors with accuracy of the level of $\sim 30 \mathrm{nrad}(\mathrm{rms})$.

\section{THE OSMS CALIBRATION SYSTEM}

Figure 2 shows the in-situ calibration system, integrated to the OSMS for calibration of the sample-arm AC. The system is based on an AC ELCOMAT-3000 (AC-3 in Figs. 1 and 2a) [53] and a custom-made tilt stage Model N-310K021 (CTS in Figs. 1 and 2a), designed and manufactured at Physik Instrumente GmbH \& Co.KG [59] especially for the application in a UTM system [60].
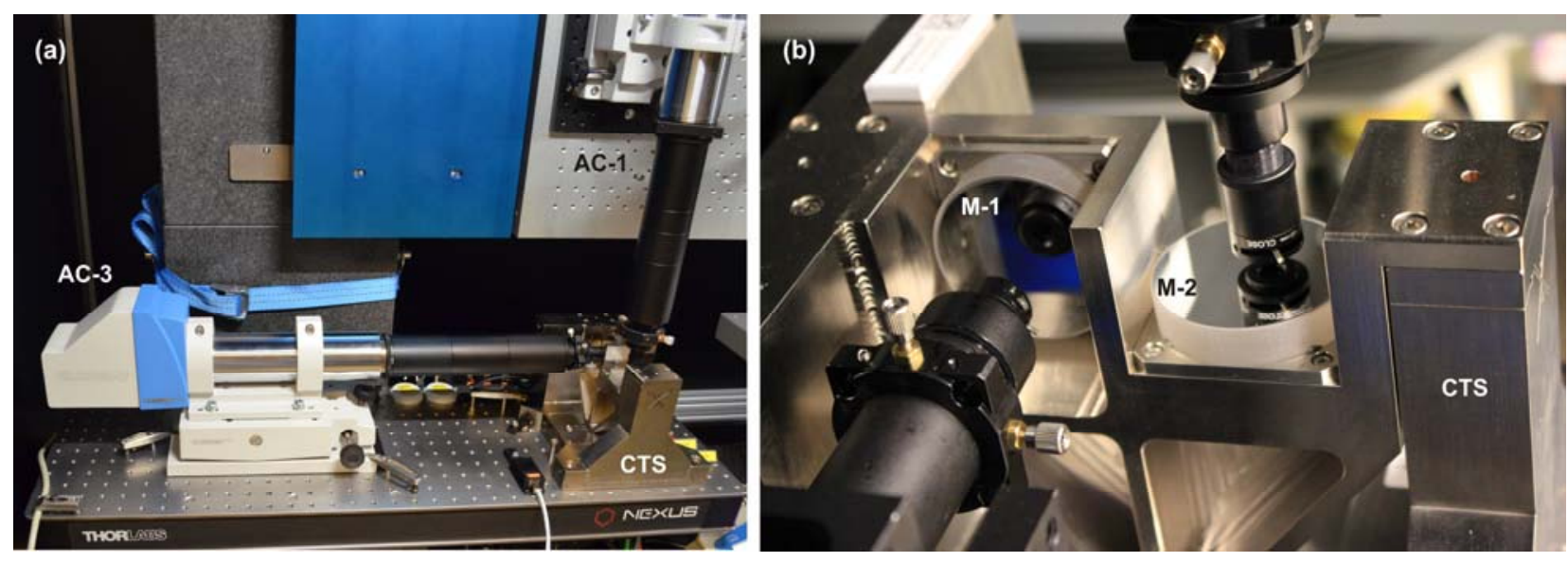

Figure 2. (a) The OSMS in-situ calibration system, based on autocollimator ELCOMAT-3000 (AC-3) [53] and the custom precision calibration tilt stage (CTS) [59], and (b) the enlarged view of the tilt stage with two reference mirrors, M-1 and $\mathrm{M}-2$, and the end parts of aperture tubes attached to the reference $\mathrm{AC}$ (AC-3) and the AC in the OSMS sample arm (AC-1).

The experimental arrangement of AC-3 in Fig. 2a, including the aperture shape, size, and distance to the AC, is as close as possible to the arrangement of the AC calibration set-up at the PTB [52]. Below, in Sec. 3.1, we present the results of the PTB calibration and discuss the limitation of its usage as a traceable reference for in-situ calibration of the AC-1 in the OSMS sample arm.

The detailed description of design and performance of the calibration tilt stage can be found in Ref. [59]. The stage precisely tilts two flat reference mirrors (M-1 and M-2 in Fig. 2b) in the range of \pm 10 mrad with angular resolution down to $4 \mathrm{nrad}$ and repeatability of about 25 (8?) nrad. The flatness of the mirrors measured over the central area of approximately 0.5 -in diameter is better that $\lambda / 100$. The mirrors are mounted to the stage with the tilt axis to lie on their reflecting surfaces. In the course of the OSMS calibration, the horizontally reflecting mirror M-1 is used for the tilt stage calibration, as discussed in Sec. 4.2, below. The vertically reflecting mirror M-2 transfers the stage calibration to the calibration of the sample-arm AC (see Sec. 4).

Therefore, the whole calibration is a three-step process and investigation of the reliability of each step is the major topic of the present work. 


\section{THE OSMS CALIBRATION METHODOLOGY}

In this section, we describe the methodology developed at the ALS XROL for precision in-situ calibration of the autocollimator AC-1 in the OSMS sample arm. The first step of the three-step calibration process is precision calibration of the AC used in the OSMS calibration system (Fig. 3a) - Sec. 4.1. In the second step (Sec. 4.2), the PTB calibration is applied to calibrate the calibration tilt stage. And finally (Sec. 4.3), the tilt stage is used to measure the systematic error of the sample-arm AC before and after a measurement with the SUT. As shown below, the reliability of each step and the total calibration process are due to the advance environmental conditions in the XROL, providing high repeatability of the OSMS measurements.

\subsection{Characterization of the OSMS calibration-reference AC at the PTB}

The AC, used currently as a calibration-reference AC (AC-3 in Figs. 1 and 2), was precisely characterized at the PTB in 2018, when the resolution performance of the AC with the beam limiting apertures of different size and diameter was investigated [52]. Here, we use the calibration of the AC, performed with a flat reference mirror, placed at a distance of $330 \mathrm{~mm}$ from the autocollimator. The distance between the AC aperture and the reference mirror was about $3 \mathrm{~mm}$.

Figures 3 and 4 present the results of the PTB calibration of the AC-3 equipped with $2.5-\mathrm{mm}$ (the red dashed lines) and 25-mm (the solid blue lines) diameter circular apertures; the variation of the AC reading error [plots (a)] and its power spectral density [PSD, plots (b)] are shown. The calibration was performed over the angular ranges of $\pm 4848 \mu \mathrm{rad}$ with steps of $48.48 \mu \mathrm{rad}$ (Fig. 3) and $\pm 97 \mu \mathrm{rad}$ with steps of $0.4848 \mu \mathrm{rad}$ (Fig. 4).
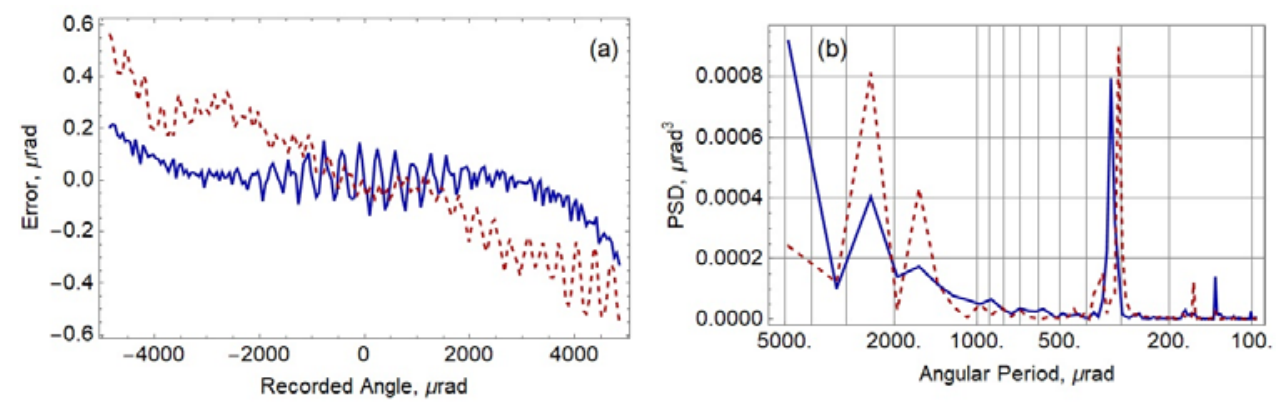

Figure 3. (a) ELCOMAT-3000 autocollimator AC-3 (Figs. 1 and 2a) calibrations with 2.5-mm-diameter circular aperture (the dashed red line) and with 25-mm-diameter aperture (the solid blue line); (b) the PSD of the calibration traces in plot (a), given as the functions of the angular period. The calibrated tangential angular range is $\pm 4848 \mu \mathrm{rad}$, sampled with $48.48-\mu \mathrm{rad}$ steps.
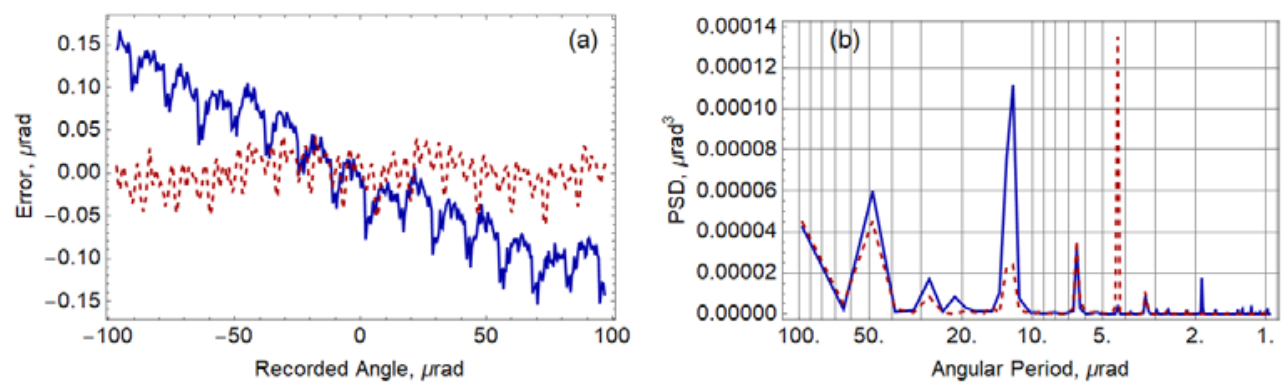

Figure 4. (a) ELCOMAT-3000 autocollimator AC-3 (Figs. 1 and 2a) calibrations with 2.5-mm-diameter circular aperture (the dashed red line) and with 25-mm-diameter aperture (the solid blue line); (b) the PSD of the calibration traces in plot (a), given as the functions of the angular period. The calibrated tangential angular range is $\pm 97 \mu \mathrm{rad}$, sampled with $0.4848-\mu \mathrm{rad}$ steps.

Besides the scalar calibration error, seen in Fig. 3a as a linear variation of the error measured with 2.5-mm aperture, and the low angular frequency variations of the error, there are a number of quasi-periodic systematic errors that are characteristic for this type of autocollimators. For a particular AC, the periods of these quasi-periodic systematic errors, depicted in the PSD distribution in Figs. 3 and 4, are the same and almost don't depend on the aperture size, even it changes by a factor of 10 . 
The largest low-angular-period oscillations of the systematic error feature a period of $\sim 315-320 \mu \mathrm{rad}$ (Fig. 3), which is related to the AC reticle's geometry [62]. The error at the lowest angular frequencies depicted in the PSD distributions in Fig. $3 \mathrm{~b}$ with peaks with periods of about $1.6 \mathrm{mrad}$ and $2.3 \mathrm{mrad}$ is possibly due to the vendor calibration routine that removes a third order polynomial.

The pixel width of the CCD detector of the ELCOMAT-3000 corresponds to an angular scale of $\sim 13 \mu \mathrm{rad}$. In the previous version of the AC ELCOMAT-3000 (that we used before with the DLTP profiler [37] at the XROL), there was a strong sawtooth-like systematic error, caused by the interaction of the reticle image with the pixels of the CCD detector and the algorithm, which determine the image shift on the detector by sub-pixel interpolation [62]. In more recent versions of the ACs, used with the OSMS, the 13- $\mu$ rad sawtooth-like systematic error is significantly suppressed. Now, the main high-angular frequency quazi-periodic error peaks in the AC error PSD in Fig. 4b is at the angular period of $4.33 \mu \mathrm{rad}$; in what could correspond to a third harmonic of $13 \mu \mathrm{rad}$. NEEDs to be verified with Ralf.

It is imperative that in the classical slope profiler arrangement with 2.5 -mm-diameter circular aperture, the overall variation of the AC systematic error is significantly larger than that of the AC with 25-mm-diameter aperture. Additionally, the calibration with the large aperture is less dependent on the environment and arrangement peculiarities of the AC application. Therefore, for the transfer of the calibration throughout this paper, we use the PTB calibration of AC-3 performed with 25-mm-diameter aperture.

\subsection{In-situ angular calibration of the tilt stage}

In spite of the high precision of the calibration in Figs. 3 and 4, performed at the PTB, direct applicability of the calibration to the measurements with the $\mathrm{AC}$, used in an experimental arrangement and conditions, different from those during the PTB calibration, is always questionable. Distance between the autocollimator and the SUT, size and shape of the beam limiting aperture, lateral aperture placement with respect to the autocollimator's optical axis, as well as environmental conditions in the lab are among the factors that dramatically affect the calibration [52,63]. The exact reproduction of the PTB calibration set-up, other than at the PTB lab, is challenging if not impossible.

For the calibration transfer, one still can use the correlation methods suggested and demonstrated in Refs. [22,44,51] for effective suppression of the quazi-periodic systematic errors of the AC. However, application of the error suppression methods requires additional measurements that makes the calibration time-consuming and less repeatable due to the slow drifts of the lab environmental conditions and the measurement set-up. Here, we describe an experimental method, which, we believe, allows the calibration tilt stage to be traceable back to the PTB angle comparator.

The method is based, first, on the experimental observation that the low angular frequency variation of the error is stable with respect to a reasonably small variation of the experimental arrangement and environmental conditions, and, second, on the natural assumption that the quazi-periodic systematic errors (Figs. 3 and 4), characteristic for the AC ELCOMAT 3000 of the calibration system, are not inherent to the tilting stage. Therefore, if the PTB calibration of the $\mathrm{AC}$ does not allow to exactly correct the AC quazi-periodic errors, an infiltration of the error to the stage calibration can be fixed by an appropriate filtering of the stage calibration data.

Figure 5 shows the raw data, after linear detrending, of the calibration of the calibration tilting stage as a dependence of the angle $\alpha_{A C}$, recorded with the AC-3 (Fig. 2a) on the input value of the CTS tilt driver, $t$.
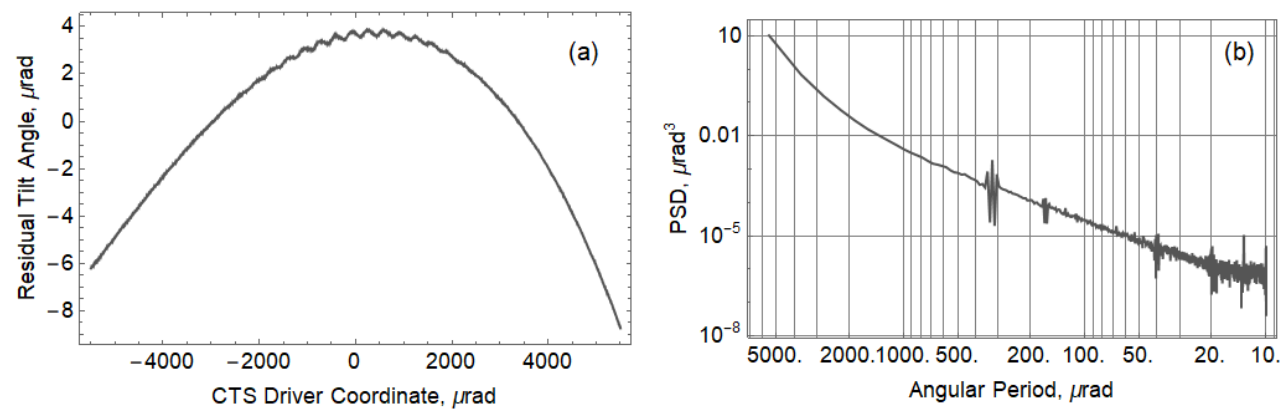

Figure 5. (a) Residual tilt angle, after detrending the best fit linear trend, measured with ELCOMAT-3000 autocollimator AC-3 with 25-mm-diameter circular aperture, as a function of the CTS tilt stage motor coordinate; (b) the PSD of the tilt angle trace in plot (a), given as the function of the angular period. The total tilt angular range is $\pm 5.5 \mathrm{mrad}$, sampled with $5 \mu \mathrm{rad}$ steps. 
The AC quasi-periodic systematic errors are seen in the PSD distribution (Fig. 5) as the spikes at the characteristic angular periods of $\sim 13 \mu \mathrm{rad}$ and $\sim 320 \mu \mathrm{rad}$. In order to better understand the magnitude of the errors, in Fig. 6 the overall trend of the dependence is detrended with a function corresponding to a simple geometrical model of the stage tilt, monitored with two encoders at the constant distance:

$$
\alpha_{\text {AC }}=A \cdot \arctan \left(B \cdot\left(t-t_{0}\right)\right)+C,
$$

where $A, B, C$, and $t_{0}$ are the fitting parameters.
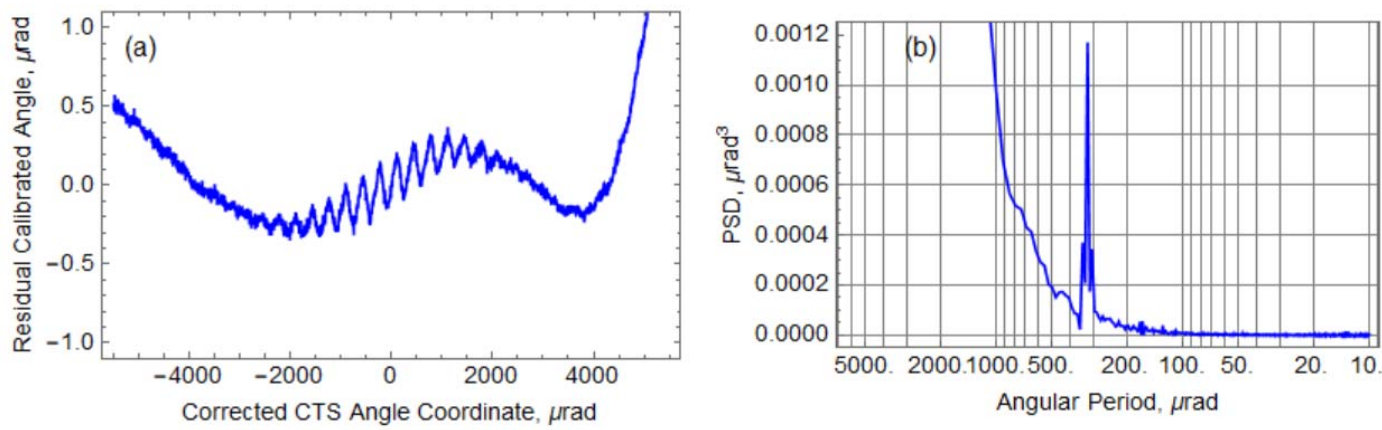

Figure 6. Angular calibration of the CTS after correction of the CTS angle coordinate [according to Eq. (1)], and detrended with the best fit linear trend: (a) residual tilt angle, measured with ELCOMAT-3000 autocollimator AC-3 with 25-mmdiameter circular aperture (no correction based on the PTB calibration), as a function of the corrected CTS angle coordinate; (b) the PSD of the tilt angle trace in plot (a), given as the function of the angular period for the corrected CTS angle coordinate. The total tilt angular range is $\pm 5.5 \mathrm{mrad}$, sampled with $5-\mu \mathrm{rad}$ steps.

Figure 5 and 6 reproduce the raw measurement, which is not corrected to account the AC calibration performed at the PTB. Application of the calibration effectively suppresses the major quasi-period error with the period of $\sim 320 \mu \mathrm{rad}-$ Fig. 7. Application of the PTB calibration also removes the low angular frequency error of the AC (Fig. 3a), corresponding to the measurements with 25 -mm-diameter aperture.
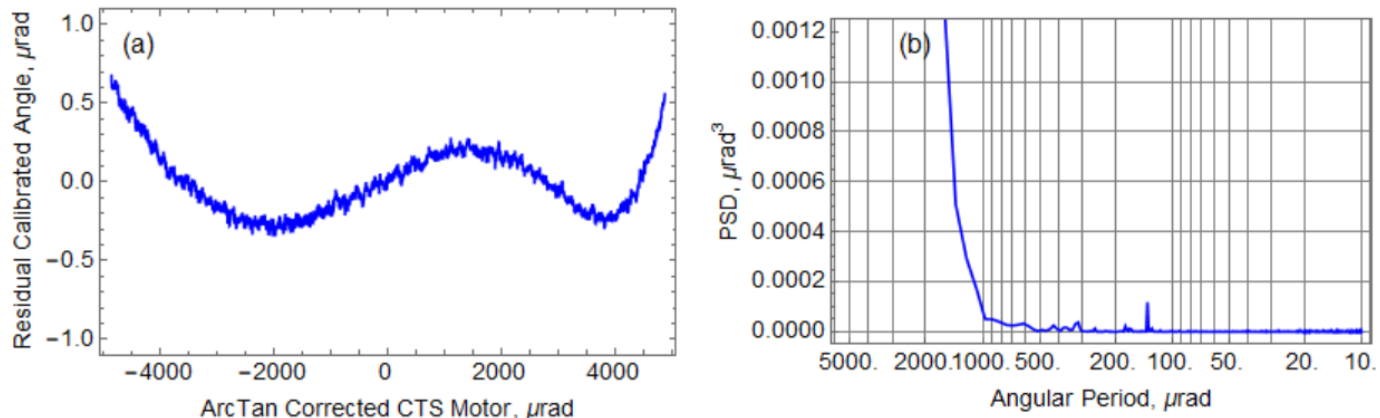

Figure 7. Angular calibration of the CTS after application of the AC calibration and correction of the CTS angle coordinate [according to Eq. (1)], and detrended with the best fit linear trend: (a) residual tilt angle, measured with ELCOMAT-3000 autocollimator AC-3 with 25-mm-diameter circular aperture and corrected with the PTB calibration data, as a function of the corrected CTS angle coordinate; (b) the PSD of the tilt angle trace in plot (a), given as the function of the angular period for the corrected CTS angle coordinate. The total tilt angular range is $\pm 5 \mathrm{mrad}$, sampled with 5 - $\mu \mathrm{rad}$ steps.

Finally, the CTS angular correction is determined by fitting the data in Fig. 7 with a series of Chebyshev polynomials. Figure 8 depicts the residual error of the fit. The applied correction and fitting lead to practically random residual error that relates to the random error of the AC measurements and setting of the tilt angle of the CTS. There is also a contribution of the aliasing effect due to sampling period different from the angular period of the 13- $\mu$ rad quasi-periodic error of the AC. For the AC-based profilometer measurements without a high-angular-resolution calibrations, the later factor sets a principle limitation to the random-looking error of the measurements [46]. 

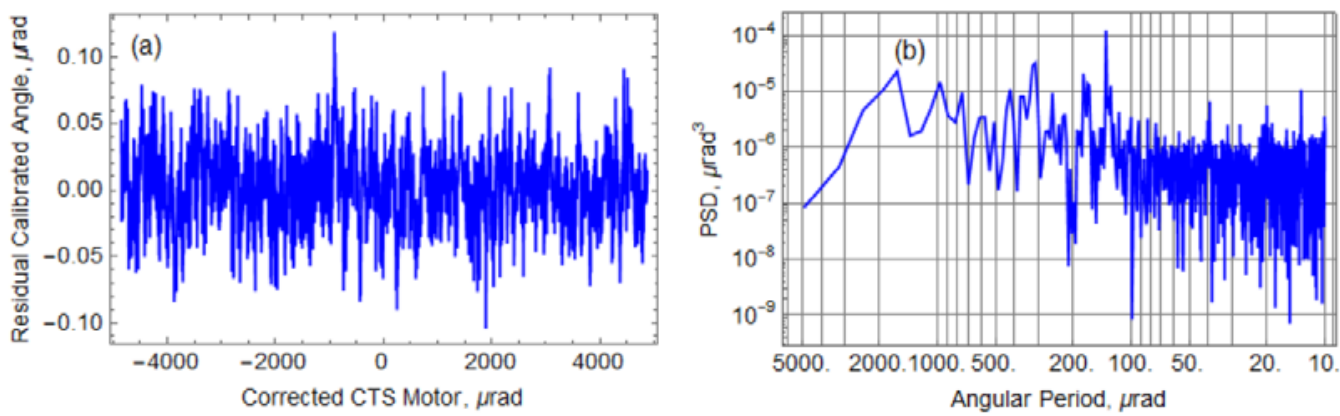

Figure 8. (a) The residual slope (after application of the AC calibration) variation obtained in the result of fitting of the CTS error in Fig. 7 with Chebyshev polynomials up to order 9 as a function of the corrected [according to Eq. (1)] CTS angle coordinate; (b) the PSD distribution of the residual error in plot (a). The total tilt angular range is $\pm 4848 \mu \mathrm{rad}$, sample with 5 - $\mu \mathrm{rad}$ steps.

In order to make the calibration free of the random error, we use the resulted fitting function (corresponding to Fig. 8) as the final CTS angular calibration function. This calibration effectively covers the high angular-period range from $500 \mu \mathrm{rad}$ to the whole angular dynamic range of the AC of $\pm 4.9 \mathrm{mrad}-$ Fig. 9.
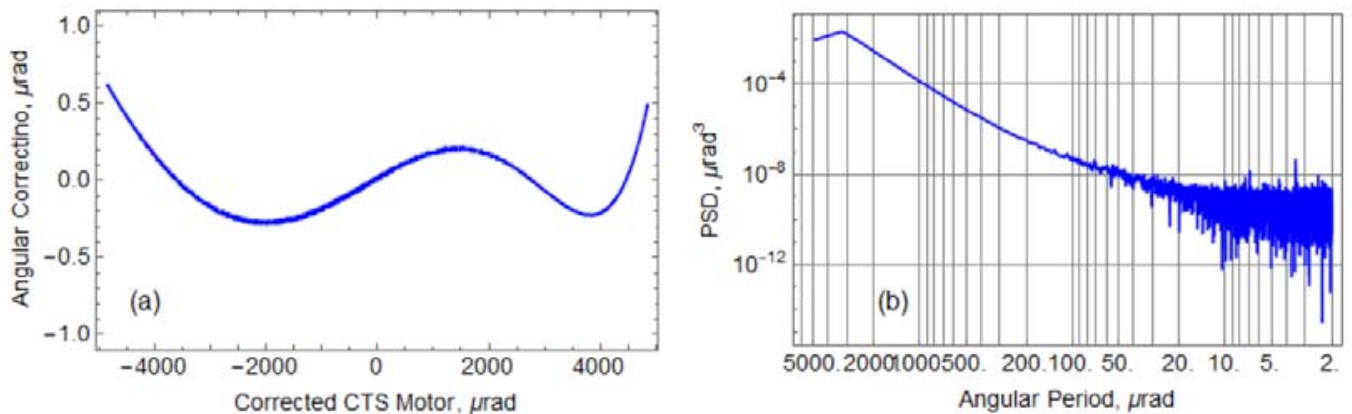

Figure 9. (a) The CTS angular error as a function of the corrected [according to Eq. (1)] CTS angle coordinate; (b) the PSD distribution of the residual error in plot (a).

\subsection{Transfer of calibration to the OSMS sample-arm AC}

With precisely calibrated CTS (Sec. 4.2), we can measure the tangential angle (AC-1 X-channel) error of the sample-arm AC. In order to ensure the applicability of the AC-1 calibration to a particular measurements with an SUT, the AC-1 calibration is performed in two runs (each with 8 scans), before and after the SUT measurements.

Figures 10 and 11 illustrate the results of the calibration runs as the average of the measured slope traces (Fig. 10) and their difference (Fig. 11) for the case of the OSMS AC-1 equipped with a circular aperture of 2.5-mm diameter.
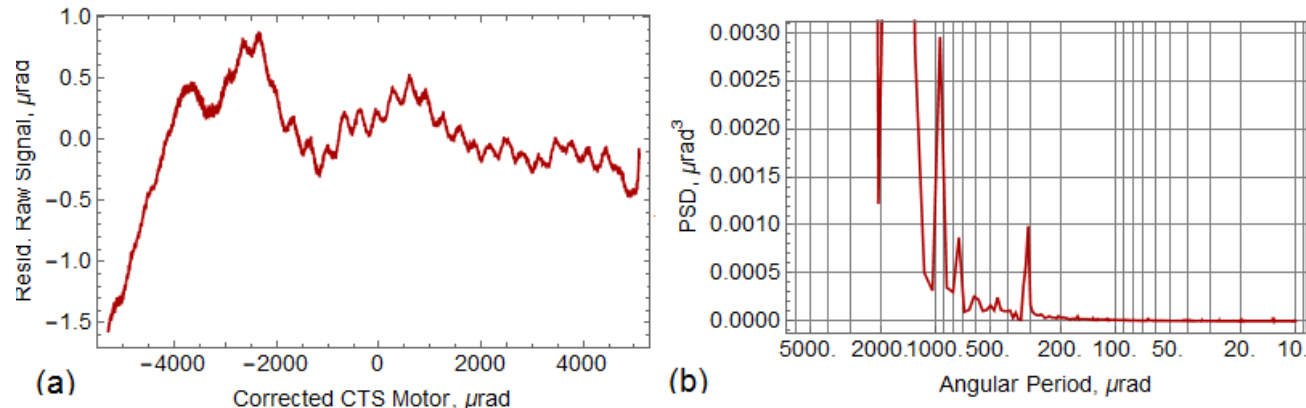

Figure 10. (a) Residual tilt angle, after detrending the best fit linear trend, of the average of the AC-1 calibration traces, measured with ELCOMAT-3000 autocollimator AC-1 with 2.5-mm-diameter circular aperture before and after the measurements with the SUT, as a function of the corrected CTS angle coordinate; (b) the PSD of the tilt angle trace in plot (a), given as the function of the angular period. The total tilt angular range is $\pm 5.5 \mathrm{mrad}$, sampled with $5 \mu \mathrm{rad}$ steps. 

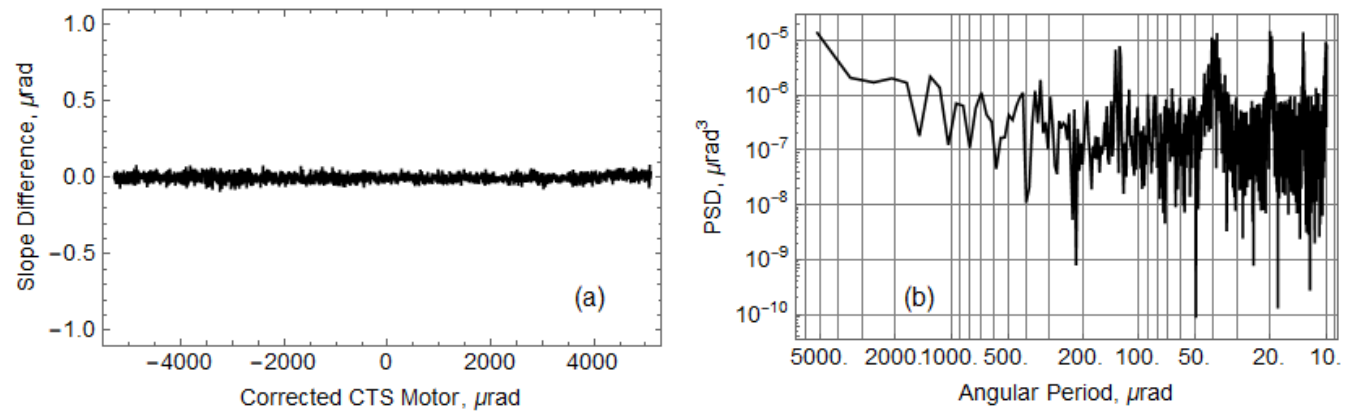

Figure 11. (a) Difference of the AC-1 calibration traces, measured with ELCOMAT-3000 autocollimator AC-1 with 2.5-mm-diameter circular aperture in two runs of 8 scans each before and after the measurements with the SUT, as a function of the corrected CTS angle coordinate; (b) the PSD of the tilt angle trace in plot (a), given as the function of the angular period. The total tilt angular range is $\pm 5.5 \mathrm{mrad}$, sampled with $5 \mu \mathrm{rad}$ steps.

The AC-1 calibration repeatability as the difference between single scans of a run performed before or after mirror measurements is characterized with $51 \mathrm{nrad} \mathrm{rms}$. Assuming almost random character of the difference (the assumption is very natural, as follows, in particular, from the almost white-noise like character of the PSD in Fig. 11), the repeatability of a singe scan withing the runs is about $36 \mathrm{nrad} \mathrm{rms}(\approx 51 / \sqrt{2} \mathrm{nrad} \mathrm{rms})$. If instead, we consider the difference of 8 -scan runs (Figs. 10 and 11$)$, the repeatability is $18.4 \mathrm{nrad} \mathrm{rms}(\approx 26 / \sqrt{2} \mathrm{nrad} \mathrm{rms})$. Finally for the average calibration, we can assume the precision on the level of about $13 \mathrm{nrad}$ rms.

Having proved the high repeatability of the calibration measurements, we can use the average of the AC-1 calibrations, determined before and after mirror measurements, and calculate the AC-1 error as a function of its angle reading. The resulted error function is shown in Fig. 12 with the bottom (solid) blue line. For comparison, the AC-1 error function measured at the PTB is depicted in Fig. 12 with the top (dashed) red line.
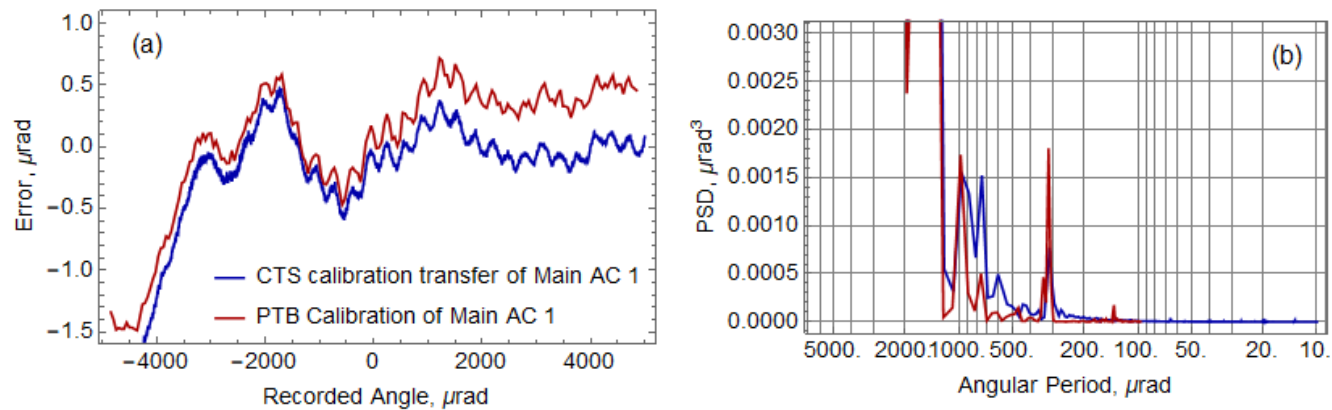

Figure 12. (a) Error functions of the OSMS ELCOMAT-3000 AC-1, equipped with 2.5-mm-diameter circular aperture, as measured at the PTB (the dashed red line) and at the ALS with the OSMS in-situ calibration system (the solid blue line); (b) the PSD of the error functions in plot (a), given as the function of the period of the recorded angle. The total tilt angular range is $\pm 5.5 \mathrm{mrad}$, sampled with $\sim 5 \mu \mathrm{rad}$ steps.

Figure 13 depicts the difference [in the angular, plot (a), and the angular wavelength, plot (b), domains] of the error functions measured at the PTB and at the ALS with in-situ OSMS calibration system (Fig. 2a). The rms variation of the difference trace is $140 \mathrm{nrad}$, mainly due to the longer-angular-period dissimilarity of the calibrations. If instead, we consider detrending a $3^{\text {rd }}$-order polynomial from the error difference, the rms variation is dropped to $\sim 44$ nrad. Note that the shorter-angular-period quasi-periodic errors are almost perfectly matched, so that the corresponding PSD peaks are hardly seen in the error difference in Fig. 13b (compare with Fig. 12b).

The observed dissimilarity of the calibrations originates from the difference of the experimental arrangements and the lab environmental conditions.

First, the PTB calibration in 2012, was performed with the distance between the AC and the reference mirror of $300 \mathrm{~mm}$, rather than the optimized value of $330 \mathrm{~mm}$, recommended in Ref. [63]. 

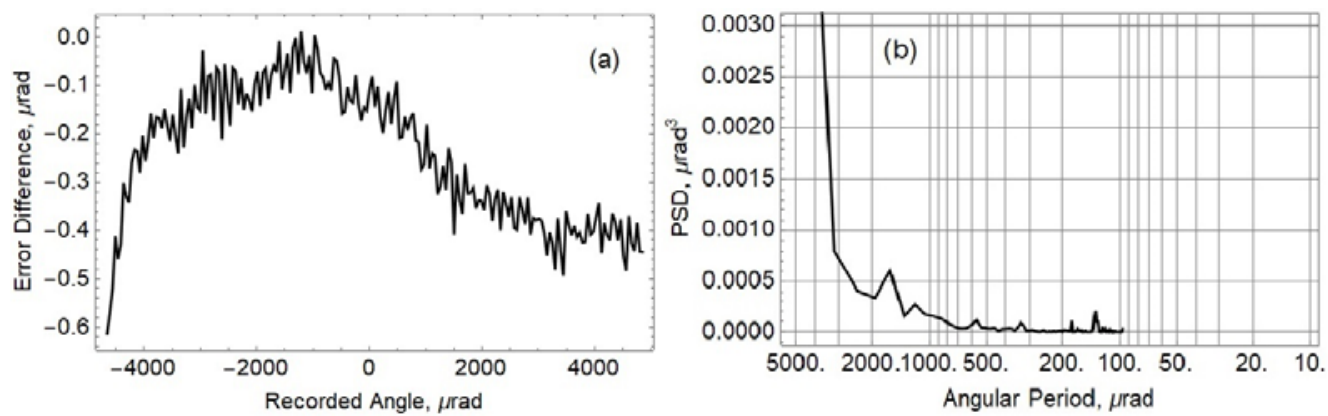

Figure 13. (a) Difference of the AC-1 error functions of the OSMS ELCOMAT-3000 AC-1, equipped with 2.5-mmdiameter circular aperture, measured at the PTB and at the ALS and shown in Fig. 12; (b) the PSD of the error difference trace in plot (a), given as the function of the angular period.

Second, in the course of calibration at the PTB, the reference mirror and the aperture device are both placed on the rotating platform of the angular comparator. This is in contrast with the usual arrangement of AC-based slope profilers (such as the XROL OSMS), where the aperture is not following to the mirror surface slope.

Third, the PTB aperture hole of 2.5 - $\mathrm{mm}$ diameter is drilled in an aluminum plate that is approximately $3 \mathrm{~mm}$ thick, whereas in the XROL OSMS setup (similar to almost all AC-based slope profilers around the world), a much thinner orifice (e.g., iris diaphragm) is usually used.

Fourth, the PTB and the XROL are using different alignment procedures discussed in detail in Ref. [64]. As we have empirically established, the direction of the light beam from a dedicated alignment laser, purchased from the $\mathrm{AC}$ vendor, is not repeatable and depends on the azimuthal position of the laser attached to the $\mathrm{AC}$ optical tube. We believe that the alignment procedure developed and routinely used at the XROL is significantly more reliable and repeatable. Unfortunately, it is not always possible to apply the procedure for aperture alignment in a setup devoted to calibration measurements (see discussion in Ref. [52]).

Firth, a subtle difference in the reflectivities of the reference mirrors, used at the PTB and at the ALS, may be an additional cause of the observed discrepancy of the error functions in Fig. 12.

In conclusion of the discussion of the calibration methodology, developed for the surfaces slope measurements with the OSMS, we should mention that it is not absolute. The major problems that remains are the differences in the reflectance and shape of the CTS reference mirror and the SUT. In order to completely overcome the problems, we are investigating the possibility for using the SUT itself as the calibration reference mirror. In principle, it seems to be feasible with the tilt-flip-Z-translation stage integrated to the OSMS gantry system (Fig. 1). The work in this direction is in progress.

\section{APPLICATION OF THE ANGULAR CALIBRATION}

In this section, we discuss the results of application of the in-situ calibration technique to the OSMS measurements with an elliptical cylinder mirror M204, designed and fabricated for the ALS QERLIN beamline 6.0.2 [65].

The gold-coated mirror with the total size of the single crystal silicon substrate of $650 \mathrm{~mm} \times 75 \mathrm{~mm} \times 50 \mathrm{~mm}$ has a clear aperture of $600 \mathrm{~mm} \times 20 \mathrm{~mm}$. The specified conjugate parameters of the mirror beamline application are following: the source distance $r=2800( \pm 10) \mathrm{mm}$, the focal (image) distance $r^{\prime}=1200( \pm 10) \mathrm{mm}$, and the grazing incidence angle $\theta=1.5( \pm 0.1)$ degrees. The mirror has a strong variation of the surface radius of curvature from $\sim 53 \mathrm{~m}$ at the downstream end to $\sim 73 \mathrm{~m}$ at the upstream range, corresponding to about $10 \mathrm{mrad}$ slope variation over the clear aperture length in the tangential direction. The specified residual slope error has to be less than $175 \mathrm{nrad}$ rms. This is after subtraction of the best-fit elliptical shape with the values of the conjugate parameters within the specified tolerances [66].

The measurements, presented in this section, were performed with the OSMS ELCOMAT-3000 AC-1, equipped with 2.5 -mm-diameter circular aperture. The measurement run of 8 sequential scans is arranged according to the AOSS technique [51]. In this case after first two scans, performed with scanning of the AC-1 along the mirror in the forward (F) and and backward (B) directions, the pitch angle of the mirror is changed by $\sim 150 \mu \mathrm{rad}$, and returned back to the original value after next four scans, arranged as a sequence of B-F-B-F. The last two scans are made with scanning in the 
forward and backward directions, F-B. The change of the pitch angle by the half of the period of the main quasi-periodic systematic error of the $\mathrm{AC}-1$ ensures strong suppression of the error to the final slope trace, resulted from the averaging all eight scans $[24,44]$. Arrangement of the scans as F-B-B-F-B-F-F-B sequence allows suppression of the drift error described with a polynomial function up to the $3^{\text {rd }}$ order [22]. Additionally, after first four scans, the OSMS automatically flips the mirror orientation by 180 degrees. Flipping the mirror orientation allows to remove from the final trace the OSMS systematic error that is even with respect to the reversal of the tangential axis $[67,68]$.

\subsection{Application of the AC calibration to a single measurement scan with the SUT}

The residual (after subtraction of the best-fit elliptical shape) slope variation of the M204 mirror, measured in a single scan of the run of 8 scans total, is presented in Figure 14. This is the raw experimental data before the AC-1 calibration is applied. As seen in the PSD in Fig. 14b, the measured slope variation has a strong contribution of the AC quasiperiodic errors with the largest error with angular period of $\sim 300 \mu \mathrm{rad}$.

(a)

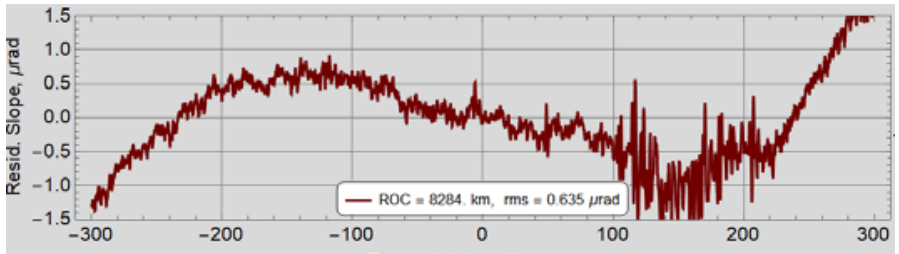

(b)

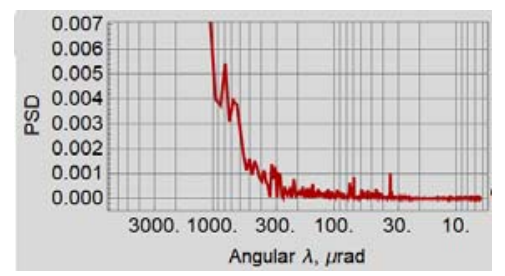

Figure 14. (a) Residual (after subtraction of the best fit elliptical shape) slope variation of the M204 mirror, as measured in a single scan of the OSMS run of 8 scans total in the case of the ELCOMAT-3000 AC-1, equipped with 2.5-mm-diameter circular aperture; there has been not applied the AC-1 calibration. (b) The PSD of the residual slope trace in plot (a).

Subtraction from the raw slope data (Fig. 14a) of the AC-1 error function, shown in Fig. 12a, suppresses the AC systematic error that is clearly seen in the Fig. 15, where the PSD peak due to the 300- $\mu$ rad quasi-periodic error is practically cleaned out (Fig. 15b). Correspondingly, the rms variation of the residual slope trace is decreasing from $\sim 635 \mathrm{nrad}$ (for the raw data in Fig. 14a) to $\sim 467 \mathrm{nrad}$ (for the calibrated data in Fig. 15a).

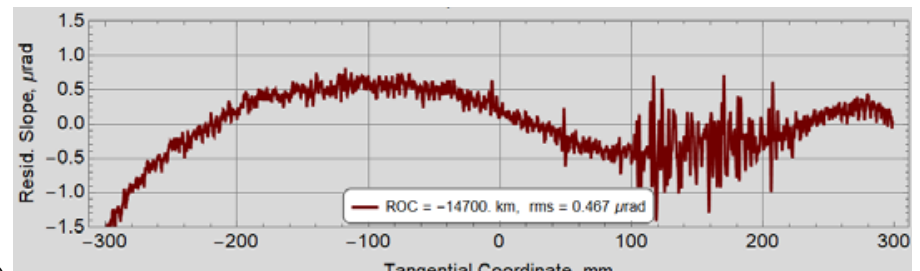

(a)

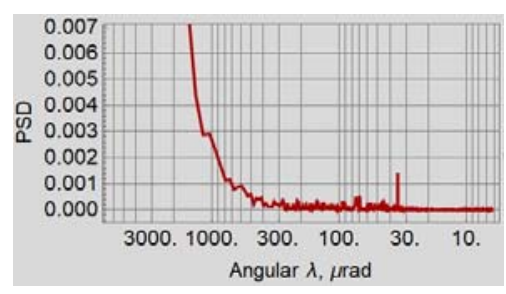

(b)

Figure 15. (a) Residual (after subtraction of the best fit elliptical shape) slope variation of the M204 mirror, as measured in a single scan of the OSMS run of 8 scans total in the case of the ELCOMAT-3000 AC-1, equipped with 2.5-mm-diameter circular aperture; there has been applied the AC-1 calibration shown in Fig. 12a. (b) The PSD of the residual slope trace in plot (a).

The applied calibration, performed with the increment of $\sim 5 \mu \mathrm{rad}$ cannot be not very efficient for suppression of the AC error with shorter periods, such as the one with the period of $\sim 13 \mu \mathrm{rad}$. If needed, this problem can be easily solved with an additional pitch tilt adjustment by $\sim 6.5 \mu \mathrm{rad}$ after each measurement scan. This reversal is orthogonal to all other reversals used in the AOSS run [51]. In case of the AC-1, this error are negligibly small (see, for example, the calibration data in Fig. 12).

Note that the quasi-periodical surface error, peaked in the PSD in Figs. $14 \mathrm{~b}$ and $15 \mathrm{~b}$ at the angular period of $\sim 35 \mu \mathrm{rad}$, is really due to the quasi-periodic error of the mirror surface with the spatial period of about $2.1 \mathrm{~mm}$. Such error is rather characteristic for some deterministic polishing processes [66,69]. In order to ensure that the error is really belong to the surface, we have performed an additional run with the mirror shifted in the tangential direction by $\sim 1 \mathrm{~mm}$ and tilted by $\sim 17 \mu \mathrm{rad}$. Additionally, by comparing the scans performed with the mirror in the direct and flipped orientation, we have convinced ourselves that the residual slope variation over the area from $100 \mathrm{~mm}$ to $200 \mathrm{~mm}$ (Fig. 15a) is also due to the slope error of the mirror surface. 


\subsection{The OSMS AC calibration method vs the advanced scanning strategy technique}

Finally, we compare the efficacy to the systematic error suppression using the methodology and experimental set-up, suggested and described here for angular calibration of the OSMS autocollimator in the sample arm, with that of the advanced scanning strategy technique [51].

Figure 16 shows the result of the OSMS run of 8 scans for measuring the QERLIN M204 mirror; there has not been applied the AC-1 calibration to this data. The same data, but with the calibration applied, are presented in Fig. 17.

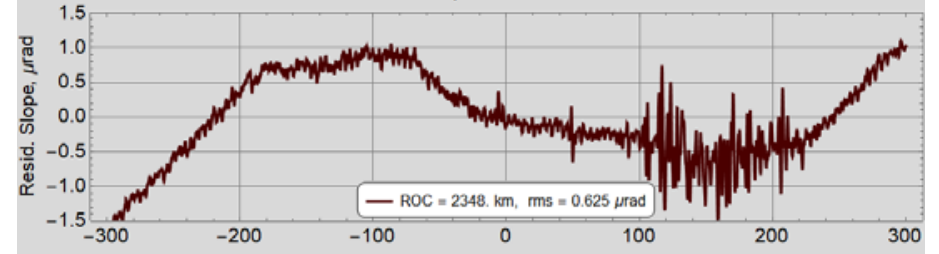

(a)

Tangential Coordinate, mm

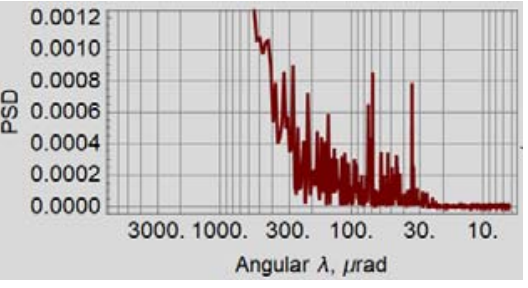

(b) Angular $\lambda, \mu \mathrm{rad}$

Figure 16. (a) Residual (after subtraction of the best fit elliptical shape) slope variation of the M204 mirror, as measured in the OSMS run of 8 scans total in the case of the ELCOMAT-3000 AC-1, equipped with 2.5-mm-diameter circular aperture; there has not been applied the AC-1 calibration. (b) The PSD of the residual slope trace in plot (a).

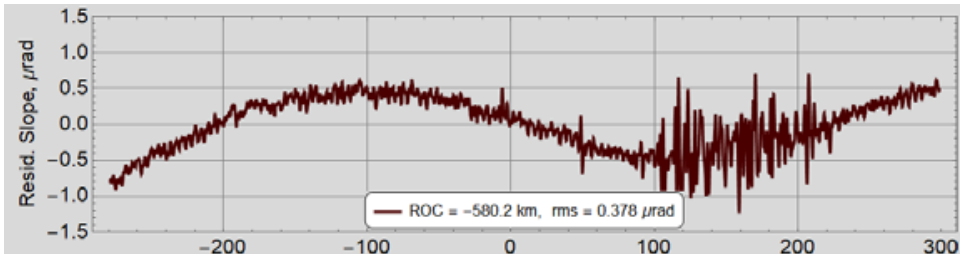

(a)

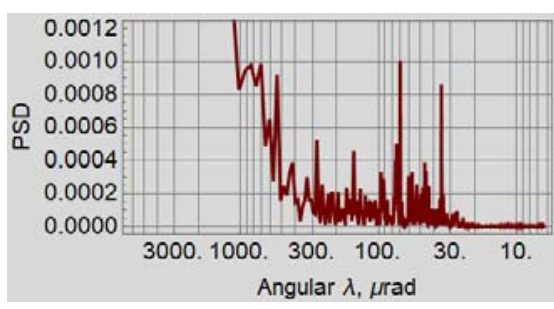

(b)

Figure 17. (a) Residual (after subtraction of the best fit elliptical shape) slope variation of the M204 mirror, as measured in the OSMS run of 8 scans total in the case of the ELCOMAT-3000 AC-1, equipped with 2.5-mm-diameter circular aperture; there has been applied the AC-1 calibration shown in Fig. 12a. (b) The PSD of the residual slope trace in plot (a).

Comparing the PSD distributions in Figs. 16b and 17b, we can conclude that the AOSS technique have the same efficacy as the in-situ calibration as to the suppression of the quasiperiodic systematic errors of the OSMS AC-1.

However, the surface slope variations at the low spatial frequency, obtained without and with application of the in-situ calibration of the AC-1 (Figs. 16a and 17a), are significantly different, characterized with the rms slope variations $625 \mathrm{nrad}$ and $378 \mathrm{nrad}$, respectively.

In order to better understand the possible contribution of the unaccounted OSMS systematic error to the result of the measurements with the M204 mirror, we can compare the difference of the slope variations resulted in the scans performed with the mirror in the direct and flipped orientations, when the calibration is not and is applied - Figs. 18 and 19, respectively. Such difference depicts the doubled even part of the systematic error that is removed by averaging of the measurements with the mirror in the direct and flipped orientations [51]. In the absence of a better estimator, one can use the rms value of the even error as a rough estimation of the possible unaccounted error, odd with respect to the reversal of the tangential axis.

If no AC-1 calibration is applied, the rms variation of the even systematic error is about 290 nrad - Fig. 18.

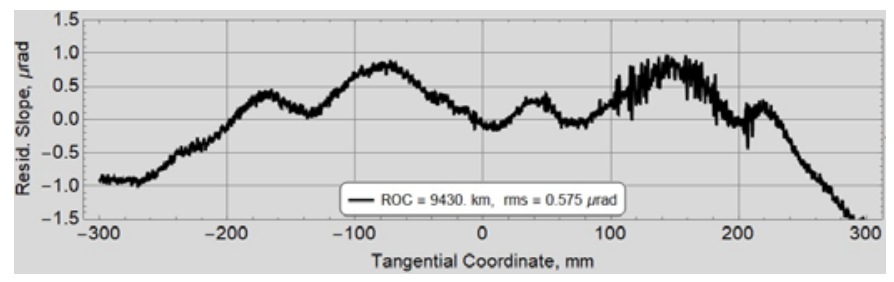

Figure 18. Difference of the slope variations resulted in the scans performed with the mirror in the direct and flipped orientation, when the calibration is not applied. The rms variation of the difference trace is about $575 \mathrm{nrad}$. 
Application of the AC-1 calibration effectively corrects the odd part of the systematic error of the measurements, decreasing it to about $65 \mathrm{mrad} \mathrm{rms}$ (Fig. 19), the value that is significantly, by a factor of almost 4, higher than the repeatability of the AC-1 calibration scans before and after the measurements with the SUT (see Sec. ).

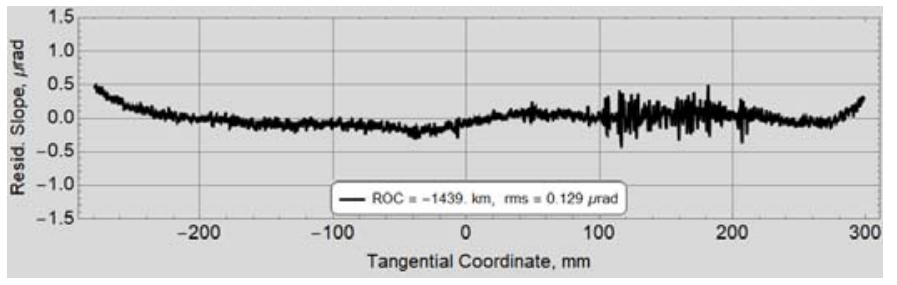

Figure 19. Difference of the slope variations resulted in the scans performed with the mirror in the direct and flipped orientation, when the calibration is applied. The rms variation of the difference trace is about 129 nrad.

Therefore, the OSMS AC calibration method and the advanced scanning strategy technique appeared to be complimentary. Application of both methods together allows us to perform the high accuracy measurements with the aspherical, long and highly curved mirror such as the QERLIN M204.

If the estimation of the unaccounted systematic error of $\sim 65 \mathrm{nrad}$ rms makes sense, the error can relate to the dependence of the AC calibration on the SUT shape. As mentioned in Sec. 4, we are investigating the possibility for using tilt-flip-Z-translation stage integrated to the OSMS gantry system to calibrate the AC-1 with the SUT as the calibration reference mirror. It seems to be feasible also because we need to better characterize the AC systematic error only over the lower spatial frequencies. The work in this direction is in progress.

\section{DISCUSSION AND CONCLUSIONS}

We have described the experimental set-up, methodology, and numerical methods developed at the ALS X-Ray Optics Laboratory for reliable transferring the angular calibration of one reference AC, performed at the PTB, to the scanning AC of the ALS Optical Surface Measuring System, an autocollimator ELCOMAT-3000 based surface slope profiler. The profiles of this type, when equipped with a $2.5-\mathrm{mm}$ circular aperture, are most commonly used for high accuracy characterization of $\mathrm{x}$-ray mirrors in the low-spatial-wavelength range, from a few millimeters to the whole length of the surface under test.

The remarkable feature of the OSMS is the movable AC (rather than a pentaprism) in the sample arm. This design ensures almost constant distance between the AC and the SUT, making the design correspondent to the arrangement of the calibration set-up at the PTB. However, we have shown that even in this case, the direct high-accuracy transfer of the PTB calibration to the OSMS is hardly possible because of the multiple differences of the experimental arrangements and conditions at the PTB and the ALS optical labs.

We have suggested and tested a new methodology for precision in-situ calibration of the OSMS sample arm AC based on a specially developed calibration system integrated to the OSMS. The first step of the three-step calibration process is precision calibration of the AC used in the OSMS calibration system. In our case, the calibration of the reference AC is performed at the PTB. In the second step, the PTB calibration is applied to calibrate the tilt stage of the calibration system. On this step, we utilize the fact that the systematic errors of the reference AC and the stage are principally different. This allows us to reliably filter out the calibration artifacts associated with the quasi-periodic systematic errors of the reference AC. And finally, the calibration tilt stage is used to measure the systematic error of the sample-arm AC before and after a measurement with the SUT.

We have discussed and demonstrated the high reliability of each step of the calibration transfer process that ensures the high accuracy of the final calibration of the OSMS. Thus, the repeatability of the calibration measurements with the sample-arm AC before and after a measurement with the SUT is about $18 \mathrm{nrad}$ rms. This became possible, in particular, due to the advance environmental conditions in the XROL.

With the example of the OSMS measurements with a highly curved aspherical mirror with a length of the clear aperture of $600 \mathrm{~mm}$, available from one of the most advanced vendor of x-ray optics, we have demonstrated the high efficacy of the developed calibration methodology and experimental methods. In this case, when the total surface slope range is about $10 \mathrm{mrad}$ (that is even slightly larger than the specified dynamic range of the ELCOMAT-3000 AC in use), the accuracy of the measurements is estimated to be on the level of $65 \mathrm{nrad} \mathrm{rms}$. 
In order to reach this level of accuracy, additionally to the angular calibration we use the advanced scanning strategy technique developed for suppression of the instrumental systematic and drift errors via multiple scans, arranged to anticorrelate the errors. Namely, the application of both methods together allows us to perform the high accuracy measurements with the aspherical, long and highly curved mirror such as the QERLIN M204.

If the estimation of the unaccounted systematic error of $\sim 65 \mathrm{nrad} \mathrm{rms,} \mathrm{made} \mathrm{in} \mathrm{this} \mathrm{paper} \mathrm{based} \mathrm{on} \mathrm{the} \mathrm{value} \mathrm{of} \mathrm{the} \mathrm{even}$ part of the systematic error removed in measurement with the mirror in the direct and flipped orientation, is valid, the error can relate to the the differences in the reflectance and shape of the CTS reference mirror and the SUT. In order to completely overcome the problems, we are investigating the possibility for using the SUT itself as the calibration reference mirror. Currently, we are investigating the possibility for using tilt-flip-Z-translation stage integrated to the OSMS gantry system to calibrate the AC-1 with the SUT as the calibration reference mirror. In principle, it seems to be feasible with the tilt-flip-Z-translation stage integrated to the OSMS gantry system, accounting that we need to better characterize the AC systematic error only over the lower spatial frequencies. The work in this direction is in progress.

\section{ACKNOWLEDGEMENTS}

Research at the Advanced Light Source at Lawrence Berkeley National Laboratory are DOE Office of Science User Facilities under contract no. DE-AC02-05CH11231.

\section{DISCLAIMER}

This document was prepared as an account of work sponsored by the United States Government. While this document is believed to contain correct information, neither the United States Government nor any agency thereof, nor The Regents of the University of California, nor any of their employees, makes any warranty, express or implied, or assumes any legal responsibility for the accuracy, completeness, or usefulness of any information, apparatus, product, or process disclosed, or represents that its use would not infringe privately owned rights. Reference herein to any specific commercial product, process, or service by its trade name, trademark, manufacturer, or otherwise, does not necessarily constitute or imply its endorsement, recommendation, or favoring by the United States Government or any agency thereof, or The Regents of the University of California. The views and opinions of authors expressed herein do not necessarily state or reflect those of the United States Government or any agency thereof or The Regents of the University of California.

\section{REFERENCES}

[1] Samoylova, L., Sinn, H., Siewert, F., Mimura, H., Yamauchi, K., and Tschentscher, T., "Requirements on hard Xray grazing incidence optics for European XFEL: Analysis and simulation of wavefront transformations," Proc. SPIE 7360, 73600E/1-9 (2009). <https://doi.org/10.1117/12.822251>.

[2] Cocco, D., "Recent Developments in UV optics for ultra-short, ultra-intense coherent light sources," Photonics 2015, 2(1), 40-49 (2015). <https://doi.org/10.3390/photonics2010040>.

[3] ALS-U, https://als.lbl.gov/als-u/.

[4] Kevan, S., Chair, [ALS-U: Solving Scientific Challenges with Coherent Soft X-Rays], Workshop report on early science enabled by the Advanced Light Source Upgrade, ALS, LBNL, Berkeley, CA, (2017). $<$ https://als.lbl.gov/wp-content/uploads/2017/08/ALS-U-Early-Science-Workshop-Report-Full.pdf $>$.

[5] von Bieren, K., "Pencil Beam Interferometer For Aspherical Optical Surfaces," Proc. SPIE 343, 101 (1982). $<$ https://doi.org/10.1117/12.933743>.

[6] von Bieren, K., "Interferometry of wave fronts reflected off conical surfaces," Appl. Opt. 22, 2109-2114 (1983). $<$ https://doi.org/10.1364/AO.22.002109>.

[7] von Bieren, K., "Pencil beam interferometer," US Patent 4,498,773 (1985).

[8] Takacs, P. Z., Qian, S., and Colbert, J., "Design of a long trace surface profiler," Proc. SPIE 749, 59-64 (1987). $<$ https://doi.org/10.1117/12.939842>.

[9] Takacs, P. Z., Feng, S. K., Church, E. L., Qian, S., and Liu, W-M., "Long trace profile measurements on cylindrical aspheres," Proc. SPIE 966, 354-64 (1989). <https://doi.org/10.1117/12.948082>.

[10] Takacs, P. Z., "X-ray Mirror Metrology," In: Bass M, editor. [Handbook of Optics V. Atmospheric Optics, Modulators, Fiber Optics, X-Ray and Neutron Optics] Vol. 5, 3rd ed: McGraw-Hill, Ch. 46 (2009). 
[11] Irick, S. C., McKinney, W. R., Lunt, D. L. T., and Takacs, P. Z., "Using a straightness reference in obtaining more accurate surface profiles," Rev. Sci. Instrum. 63, 1436-1438 (1992). <https://doi.org/10.1063/1.1143036>.

[12] Irick, S. C., "Improved measurement accuracy in a long trace profiler: compensation for laser pointing instability," Nucl. Instrum. Methods Phys. Res. A 347, 226-230 (1994). <https://doi.org/10.1016/0168-9002(94)91882-1>.

[13] Takacs, P. Z., Church, E. L., Bresloff, C. J., Assoufid, L., "Improvements in the accuracy and the repeatability of long trace profiler measurements," Appl. Optics 38(25), 5468-5479 (1999). $<$ https://doi.org/10.1364/AO.38.005468>.

[14] Qian, S. N., Takacs, P., Sostero, G., and Cocco, D., "Portable long trace profiler: Concept and solution," Rev. Sci. Instrum. 72(8), 3138-3204 (2003). <https://doi.org/10.1063/1.1384452>.

[15] Cocco, D., Sostero, G., and Zangrando, M., "Technique for measuring the groove density of diffraction gratings using the long trace profiler," Rev. Sci. Instrum. 74(7), 3544-3548 (2003). <https://doi.org/10.1063/1.1584080>.

[16] Rommeveaux, A., Hignette, O., and Morawe, C., "Mirror metrology and bender characterization at ESRF," Proc. SPIE 5921, 59210N/1-8 (2005). <https://doi.org/10.1117/12.621379>.

[17] Yashchuk, V. V., "Positioning errors of pencil-beam interferometers for long-trace profilers," Proc. SPIE 6317, 63170A/1-12 (2006). <https://doi.org/10.1117/12.677956>.

[18] Yashchuk, V. V., Irick, S. C., MacDowell, A. A., McKinney, W. R., and Takacs, P. Z., "Air convection noise of pencil-beam interferometer for long-trace profiler," Proc. SPIE 6317, 63170D/1-12 (2006). $<$ https://doi.org/10.1117/12.681297>.

[19] Kirschman, J. L., Domning, E. E., McKinney, W. R., Morrison, G. Y., Smith, B. V., and Yashchuk, V. V., "Performance of the upgraded LTP-II at the ALS Optical Metrology Laboratory," Proc. SPIE 7077, 70770A/1-12 (2008). <https://doi.org/10.1117/12.796335>.

[20] Rommeveaux, A., Thomasset, M., and Cocco, D., “The Long Trace Profilers," in [Modern Developments in X-ray and Neutron Optics], A. Erko, M. Idir, T. Krist, A. G. Michette, Eds., Chapter 10, Springer-Verlag, Berlin/Heidelberg (2008).

[21] Thomasset M. and Polack, F., "Characterization of optical surfaces for the present generations of synchrotron sources," Proc. SPIE 7155, 715506/1-12 (2008). <https://doi.org/10.1117/12.814695>.

[22] Yashchuk, V. V., "Optimal Measurement Strategies for Effective Suppression of Drift Errors,” Rev. Sci. Instrum. 80, 115101/1-10 (2009). < http://dx.doi.org/10.1063/1.3249559>.

[23] Senba, Y., Kishimoto, H., Ohashi, H., Yumoto, H., Zeschke, T., Siewert, F., Goto, S., Ishikawa, T., "Upgrade of long trace profiler for characterization of high-precision X-ray mirrors at SPring-8," Nucl. Instrum. and Methods A 616(2-3), 237-240 (2010). <https://doi.org/1010.1016/j.nima.2009.12.007>.

[24] Ali, Z., Artemiev, N. A., Cummings, C. L., Domning, E. E., Kelez, N., McKinney, W. R., Merthe, D. J., Morrison, G. Y., Smith, B. V., and Yashchuk, V. V., "Automated suppression of errors in LTP-II slope measurements with Xray optics," Proc. SPIE 8141, 81410O-1-15 (2011). <https://doi.org/10.1117/12.894061>.

[25] Qian, J., Sullivan, J., Erdmann, M., and Assoufid, L., "Performance of the APS optical slope measuring system," Nucl. Instr. and Meth. A 710, 48-51 (2013). <https://doi.org/10.1016/j.nima.2012.10.102>.

[26] Senba, Y., Kishimoto, H., Miura, T., and Ohashi, H., "Development of a long trace profiler at SPring-8 using a newly developed slope sensor," Proc. SPIE 9962, 996204 (2016). <https://doi.org/10.1117/12.2239394>.

[27] Centers, G., Smith, B. V., and Yashchuk, V. V., "New operational mode of the pencil beam interferometry based LTP,” Proc. SPIE 9962, 996202/1-13 (2016). < https://doi.org/10.1117/12.2238298>.

[28] Nikitin, S. M., Gevorkyan, G. S., McKinney, W. R., Lacey, I., Takacs, P. Z., and Yashchuk, V. V., "New twist in the optical schematic of surface slope measuring long trace profiler," Proc. SPIE 10388, 103850I-1-17 (2017). $<$ https://doi.org/10.1117/12.2274400>.

[29] Thomasset, M., Dvorak, J., Brochet, S., Dennetiere, D., and Polack, F., "Grating metrology for X-ray and V-UV synchrotron beamlines at SOLEIL,” Rev. Sci. Instrum. 90(2), 021714 (2019). <https://doi.org/10.1063/1.5055284>.

[30] Lin, S.-W., Wang, D.- J., Fu, H.-W., Tsai, H. M., Hua, C.-Y., Kuo, C.-Y., Hsu, M. -Y., Kao, K. -Y., Yin, G.-C., Fung, H.-S., Perng, S. -Y., Chang, C.-F., "Development of a long trace profiler in situ at National Synchrotron Radiation Research Center," Rev. Sci. Instrum. 90(2), 021716 (2019) < https://doi.org/10.1063/1.5055634>.

[31] Yashchuk, V. V., Lacey, I., Arnold, T, Paetzelt, H., Rochester, S., Siewert, F., and Takacs, P. Z., "Investigation on lateral resolution of surface slope profilers," Abstract to SPIE Optics + Photonics 2019, Conference OP313: Advances in Metrology for X-Ray and EUV Optics VIII (San Diego, CA 11-15 August); proceeding tracking number: $11109-28$ 
[32] Siewert, F., Noll, T., Schlegel, T., Zeschke, T., and Lammert, H., “The Nanometer Optical Component Measuring machine: a new Sub-nm Topography Measuring Device for X-ray Optics at BESSY," AIP Conf. Proc. 705, 847850 (2004). < https://doi.org/10.1063/1.1757928>.

[33] Siewert, F., Buchheim, J., and Zeschke, T., "Characterization and calibration of 2nd generation slope measuring profiler,” Nucl. Instrum. and Meth. A 616(2-3), 119-127 (2010). <https://doi.org/10.1016/j.nima.2009.12.033>.

[34] Siewert, F., Buchheim, J., Boutet, S., Williams, G. J., Montanez, P. A., Krzywinski, J., and Signorato, R., "Ultraprecise characterization of LCLS hard X-ray focusing mirrors by high resolution slope measuring deflectometry," Opt. Express 20(4), 4525-4536 (2012). <https://doi.org/10.1364/OE.20.004525>.

[35] Geckeler, R. D. and Just, A., "Optimized use and calibration of autocollimators in deflectometry,” Proc. SPIE 6704, 670407/1-12 (2007). <https://doi.org/10.1117/12.732384>.

[36] Alcock, S. G., Sawhney, K. J. S., Scott, S., Pedersen, U., Walton, R., Siewert, F., Zeschke, T., Senf, F., Noll, T., and Lammert, H., "The Diamond-NOM: A non-contact profiler capable of characterizing optical figure error with subnanometre repeatability," Nucl. Instr. Meth. A 616(2-3), 224-228 (2010). $<$ https://doi.org/10.1016/j.nima.2009.10.137>.

[37] Yashchuk, V. V., Barber, S., Domning, E. E., Kirschman, J. L., Morrison, G. Y., Smith, B. V., Siewert, F., Zeschke, T., Geckeler, R., and Just, A., "Sub-microradian surface slope metrology with the ALS Developmental Long Trace Profiler," Nucl. Instr. and Meth. A 616(2-3), 212-223 (2010). <https://doi.org/10.1016/j.nima.2009.10.175>.

[38] Yashchuk, V. V., "Sub-microradian surface slope metrology at the ALS Optical Metrology Laboratory and around the World," Oral presentation at the First Meeting on Development of a New Optical Surface Slope Measuring System - OSMS-I (ALS, Berkeley, March 26, 2010).

[39] Barber, S. K., Morrison, G. Y., Yashchuk, V. V., Gubarev, M. V., Geckeler, R. D., Buchheim, J., Siewert, F., and Zeschke, T., "Developmental long trace profiler using optimally aligned mirror based pentaprism," Opt. Eng. 50(5), 053601/1-10 (2011). <https://doi.org/10.1117/1.3572113>.

[40] Barber, S. K., Geckeler, R. D., Yashchuk, V. V., Gubarev, M. V., Buchheim, J., Siewert, F., and Zeschke, T., "Optimal alignment of mirror based pentaprism for scanning deflectometric devices," Opt. Eng. 50(7), 0073602-1-8 (2011). <https://doi.org/10.1117/1.3598325>.

[41] Nicolas, J. and Martınez, J. C., "Characterization of the error budget of Alba-NOM," Nucl. Instr. and Meth. A 710, 24-30 (2013). <https://doi.org/10.1016/j.nima.2012.10.125>.

[42] Assoufid, L., Brown, N., Crews, D., Sullivan, J., Erdmann, M., Qian, J., Jemian, P., Yashchuk, V. V., Takacs, P. Z., Artemiev, N. A., Merthe, D. J., McKinney, W. R., Siewert, F., and Zeschke, T., "Development of a highperformance gantry system for a new generation of optical slope measuring profilers," Nucl. Instrum. Meth. A 710, 31-36 (2013). <https://doi.org/10.1016/j.nima.2012.11.063>.

[43] Siewert, F., Buchheim, J., Höft, T., Zeschke, T., Schindler, A., and Arnold, T., "Investigations on the spatial resolution of autocollimator-based slope measuring profilers," Nucl. Instrum. Methods A 710, 42-47 (2013). $<$ https://doi.org/10.1016/j.nima.2012.10.130>.

[44] Yashchuk, V. V., Artemiev, N. A., Lacey, I., and Merthe, D. J., "Correlation analysis of surface slope metrology measurements of high quality x-ray optics,” Proc. SPIE 8848, 88480I/1-15 (2013). $<$ https://doi.org/10.1117/12.2024694>.

[45] Lacey, I., Artemiev, N. A., Domning, E. E., McKinney, W. R., Morrison, G. Y., Morton, S. A., Smith, B. V., and Yashchuk, V. V., "The developmental long trace profiler (DLTP) optimized for metrology of side-facing optics at the ALS,” Proc. SPIE 9206, 920603 (2014). < https://doi.org/10.1117/12.2061969>.

[46] Qian, S., Geckeler, R. D., Just, A., Idir, M., and Wu, X., "Approaching sub-50 nanoradian measurements by reducing the saw-tooth deviation of the autocollimator in the Nano-Optic-Measuring Machine," Nucl. Instr. and Meth. A 785, 206-212 (2015). <https://doi.org/10.1016/j.nima.2015.02.065>.

[47] Qian, S. and Idir, M., "Innovative nano-accuracy surface profiler for sub-50 nrad rms mirror test," Proc. SPIE 9687, 96870D (2016). <https://doi.org/10.1117/12.2247575>.

[48] Siewert, F., Zeschke, T., Arnold, T., Paetzeld, H., and Yashchuk, V. V., "Linear chirped slope profile for spatial calibration in slope measuring deflectometry," Rev. Sci. Instrum. 87(5), 051907/1-8 (2016). $<$ https://doi.org/10.1063/1.4950737>.

[49] Lacey, I., Adam, J., Centers, G., Gevorkyan, G. S., Nikitin, S. M., Smith, B. V., and Yashchuk, V. V., "Development of a high performance surface slope measuring system for two-dimensional mapping of x-ray optics,” Proc. SPIE 10385, 103850G (2017). <https://doi.org/10.1117/12.2273029>.

[50] Lacey, I., Anderson, K., Centers, G. P., Geckeler, R. D. Gevorkyan, G. S., Just, A., Nicolot, T., Smith, B. V., and Yashchuk, V. V., "The ALS OSMS: Optical Surface Measuring System for high accuracy two-dimensional slope 
metrology with state-of-the-art x-ray mirrors," Proc. SPIE 10760, 1076002 (2018).

$<$ https://doi.org/10.1117/12.2321347>.

[51] Yashchuk, V. V., Centers, G., Gevorkyan, G. S., Lacey, I., and Smith, B. V., "Correlation methods in optical metrology with state-of-the-art x-ray mirrors," Proc. SPIE 10612, 106120O (2018). $<$ https://doi.org/10.1117/12.2305441>.

[52] Lacey, I., Geckeler, R. D., Just, A., Siewert, F., Arnold, T., Paetzelt, H., Smith, B. V., and Yashchuk, V. V., "Optimization of size and shape of aperture in autocollimator-based deflectometric profilometers," Rev. Sci. Instrum. 90(2), 021717 (2019). <https://doi.org/10.1063/1.505871>.

[53] MÖLLER-WEDEL OPTICAL， GmbH， "ELCOMAT 3000.” <https://www.haag-streit.com/moeller-wedeloptical/products/electronic-autocollimators/elcomat-series/elcomat-3000/>.

[54] Probst, R., Wittekopf, R., Krause, M., Dangschat, H., and Ernst, A., “The new PTB angle comparator,” Meas. Sci. Technol. 9, 1059 - 1066 (1998).

[55] Just, A., Krause, M., Probst, R., Bosse, H., Haunerdinger, H., Spaeth, C., Metz, G., and Israel, W., "Comparison of angle standards with the aid of a high-resolution angle encoder," Precis. Eng. 33(4), 530-3 (2009).

[56] Geckeler, R. D., Link, A., Krause, M., and Elster, C., "Capabilities and limitations of the self-calibration of angle encoders," Meas. Sci. Technol. 25, 055003/1-10 (2014).

[57] Yashchuk, V. V., Artemiev, N. A., Lacey, I., McKinney, W. R., and Padmore, H. A., "Advanced environmental control as a key component in the development of ultra-high accuracy ex situ metrology for x-ray optics," Opt. Eng. 54(10), 104104/1-14 (2015). <https://doi.org/10.1117/1.OE.54.10.104104>.

[58] Yashchuk, V. V., Artemiev, N. A., Lacey, I., McKinney, W. R., and Padmore, H. A., "A new X-ray optics laboratory (XROL) at the ALS: Mission, arrangement, metrology capabilities, performance, and future plans," Proc. SPIE 9206, 92060I/1-19 (2014). <https://doi.org/10.1117/12.2062042>.

[59] Yashchuk, V. V., Artemiev, N. A., Centers, G., Chaubard, A., Geckeler, R. D., Lacey, I., Marth, H., McKinney, W. R., Noll, T., Siewert, F., Winter, M., and Zeschke, T., "High precision tilt stage as a key element to universal test mirror for characterization and calibration of slope measuring instruments," Rev. Sci. Instrum. 87(5), 051904 (2016). <https://doi.org/10.1063/1.4950729>.

[60] Yashchuk, V. V., McKinney, W. R., Warwick, T., Noll, T., Siewert, F., Zeschke, T., and Geckeler, R. D., "Proposal for a Universal Test Mirror for Characterization of Slope Measuring Instruments," Proc. SPIE 6704, 67040A/1-12 (2007). <https://doi.org/10.1117/12.732719>.

[61] Yashchuk, V. V., Rochester, S., Lacey, I., and Babin, S., "Beyond-resolution reconstruction of 1D surface slope topography based on measured instrument's optical transfer function," Opt. Express (submitted: July 03, 2019).

[62] Geckeler, R., Just, A., Krause, M., Yashchuk, V. V., “Autocollimators for deflectometry: Current status and future Progress," Nucl. Instr. and Meth. A 616, 140-146 (2010). <https://doi.org/10.1016/j.nima.2009.11.021>.

[63] Geckeler, R. D., Křen, P., Just, A., Schumann, M., Krause, M., and Yashchuk, V. V., "Environmental influences on autocollimator-based angle and form metrology,” Rev. Sci. Instrum. 90(2), 021705/1-15 (2019). $<$ https://doi.org/10.1063/1.5057402>.

[64] Geckeler, R. D., Artemiev, N. A., Barber, S. K., Just, A., Lacey, I., Kranz, O., Smith, B. V., and Yashchuk, V. V., "Aperture alignment in autocollimator-based deflectometric profilometers," Rev. Sci. Instrum. 87(5), 051906/1-8 (2016). <doi: 10.1063/1.4950734>.

[65] Chuang, Y.-D., Anderson, C., Benk, M., Goldberg, K., Voronov, D., Warwick, T., Yashchuk, V., and Padmore, H. A., "Multiplexed high resolution soft x-ray RIXS," AIP Conf. Proc. 1741, 050011/1-5 (2016). <doi: 10.1063/1.4952931>

[66] Yashchuk, V. V., Lacey, I., Gevorkyan, G. S., McKinney, W. R., Smith, B. V., and Warwick, T., "Ex situ metrology of aspherical pre-shaped x-ray mirrors at the Advanced Light Source," Rev. Sci. Instrum. 90(2), 021711/1-13 (2019). <doi: 10.1063/1.5057441>.

[67] McKinney, W. R. and Irick, S. C. "XUV synchrotron optical components for the Advanced Light Source: Summary of the requirements and the developmental program," Proc. SPIE. 1740, 154-160 (1993). <doi: $10.1117 / 12.138697>$.

[68] Irick, S. C. "Error Reduction Techniques for Measuring Long Synchrotron Mirrors," Proc. SPIE 3447, 101-108 (1998). <doi: 10.1117/12.331122>.

[69] Siewert, F., Buchheim, J., Zeschke, T., Stormer, M., Falkenberg, G., and Sankari, R., "On the characterization of ultra-precise X-ray optical components: advances and challenges in ex situ metrology,” J. Synchrotron Rad. 21, 968-975 (2014). <doi: 10.1107/S1600577514016221>. 